\title{
Psychology of Craving
}

\author{
Sushil Sharma ${ }^{1}$, Bal Nepal ${ }^{2}$, Carolyn S. Moon $^{1}$, Anthony Chabenne ${ }^{1}$, Azza Khogali ${ }^{1}$, Comfort Ojo ${ }^{1}$, \\ Esther Hong ${ }^{1}$, Rochelle Gaudet ${ }^{1}$, Ali Sayed-Ahmad ${ }^{1}$, Amanda Jacob ${ }^{1}$, Mujtaba Murtuza ${ }^{1}$, \\ Michelle Firlit ${ }^{1}$ \\ ${ }^{1}$ Saint James School of Medicine, Anguilla Sub-Campus, Bonaire, The Netherlands \\ ${ }^{2}$ School of Public Health, University of Texas Health Sciences at Houston, Houston, USA \\ Email: *Sharma@mail.sjsm.org
}

Received October 23, 2013; revised November 23, 2013; accepted December 1, 2013

Copyright (c) 2014 Sushil Sharma et al. This is an open access article distributed under the Creative Commons Attribution License, which permits unrestricted use, distribution, and reproduction in any medium, provided the original work is properly cited. In accordance of the Creative Commons Attribution License all Copyrights (C) 2014 are reserved for SCIRP and the owner of the intellectual property Sushil Sharma et al. All Copyright (C) 2014 are guarded by law and by SCIRP as a guardian.

\section{ABSTRACT}

Craving is a complex psychological condition characterized by impaired dietary intake, sleep disturbance, sexual dysfunction, and drug abuse. Craving may be associated with risk-taking reckless behavior to seek pleasure including cigarette smoking, binge alcohol drinking, and illicit drug abuse (e.g. cocaine, methamphetamine, methylene deoxy meth-amphetamine (MDMA), ecstasy, morphine and heroine). Craving for food, sex and drugs involves the limbic system and prefrontal cortex. Thus, basic understanding of craving is exceedingly important for the better clinical management of major depression, hopelessness, and poor quality of life among young adolescents, as well as morbidity and early mortality among adults. Physical, psychological, nutritional, and medical rehabilitation may be helpful in the effective clinical management of patients with craving of any age, sex and race. Further studies on the psychology of craving will curb the number of hospital admissions as several victims of drug craving develop schizophrenia later in their life, if they remain untreated.

\section{KEYWORDS}

Craving; Drug Seeking Behavior; Limbic System; Prefrontal Cortex; Schizophrenia; Morbidity; Mortality; Drug Addiction; Psychotherapy; Physical and Nutritional Rehabilitation; Charnoly Body

\section{Introduction}

Psychological dependence and drug addiction are two distinct entities. Drug addiction involves reversible or irreversible structural, functional, and biochemical modifications in the prefrontal cortex and the limbic system. In addition, it involves entry of chemical substances through the blood brain barrier which can induce neurobehavioral and neuromorphological changes in the brain regional neurocircuitry implicated in drug seeking behavior. Psychological dependence does not involve ireversible structural, functional, and neurochemical modifications as the integrity of the blood brain barrier remains intact in these cases [1,2]. Patients with craving for drugs of abuse may be trapped in a vicious circle of habituation, morbidity, and early mortality. They may require psychotherapy, chemotherapy, or both, to accom-

"Corresponding author. plish better clinical prognosis particularly among those with anorexia nervosa and suicidal tendencies. Psychological support and nutritional rehabilitation by providing $\gamma$-sterilized food at cryogenic temperature (Radapertized; Radurized) may boost their immune system and enhance their desire for good quality food, proper sleep, and sex habits, thereby improving their quality of life [3].

It is now well known that several victims with drug abuse can develop schizophrenia in their later life [4]. There are two distinct categories of craving. The first is craving to seek survival such as in Kwashiorkor and marasmus. The second type of craving is to seek pleasure irrespective of the life-threatening consequences. A subject with craving for drugs of abuse may experience early morbidity and mortality with poor quality of life. Hence a basic understanding of food (usually high salt intake, carbohydrate and fat-rich) addiction, sex addiction, and drug addiction will provide better clinical management of 
these patients. Further study in this direction will provide better treatment options to deal with depression, lethargy, and poor quality of life in these patients. In the present article, we have discussed the clinical psychology of craving with special emphasis on food, sex and drug addiction.

\section{Vicious Circle of Addiction}

Addiction can be broadly classified into four major phases: 1) Desire, 2) Craving, 3) Rush, and 4) Crash. During the crash phase, a victim can indulge in any lifethreatening event even if he/she is aware of the deleterious consequences. Addiction could be related to a particular type of food, sex, and drugs to seek pleasure. If it remains uncontrolled, it may cause physical tolerance and dependence leading to early morbidity and mortality. The various phases of craving for drug addiction are illustrated in Figure 1.

\section{Psychological Dependence}

Psychological dependence is a type of craving for junk food containing high salt, fat and carbohydrates in addition to gambling, pornography, shopping, spending, work, and computer abuse [5]. All these events are usually not associated with any permanent structural and functional alterations in the blood brain barrier and may be considered as temporary psychological dependence. However cigarette smoking, binge alcohol drinking, illicit drug abuse involves crossing of chemical substances through the blood brain barrier (BBB). These substances may induce neuroadaptation by altering brain regional neurocircuitry involving learning, intelligence, memory, and behavior impairments as illustrated in Figure 2.

\section{Neurobiology of Addiction}

The neurobiology of addiction involves the limbic system and the prefrontal cortex. PET neuroimaging with

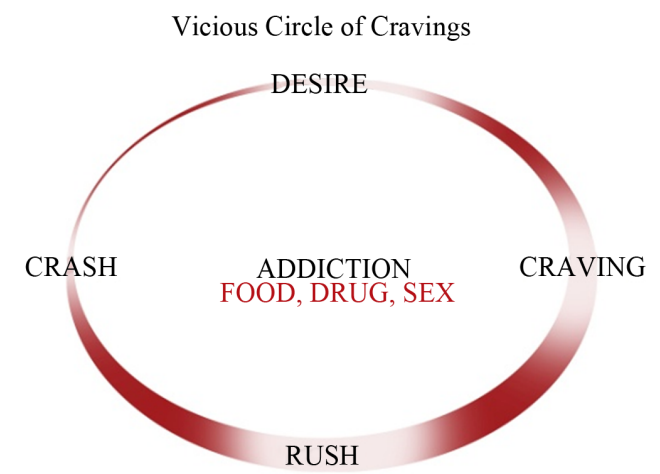

Figure 1. A diagram illustrating four different phases of drug, sex, and food addiction: 1) Desire, 2) Craving, 3) Rush, and 4) Crash.

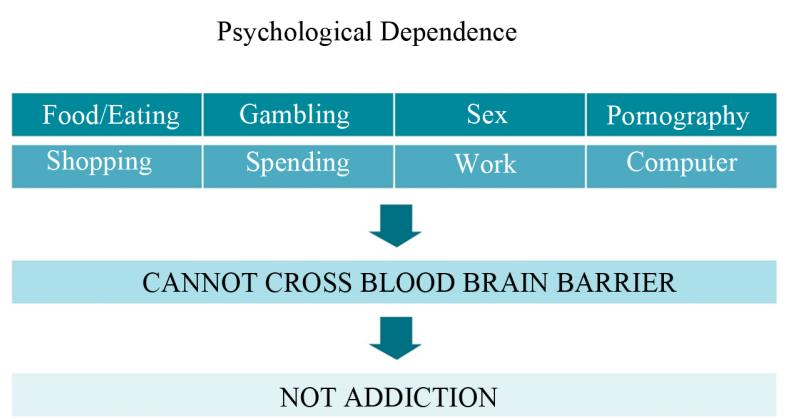

Figure 2. A diagram illustrating various types of psychological dependence which involves no entry of chemical substances through the blood brain barrier and may be considered mild craving of reversible nature.

${ }^{18} \mathrm{FdG}$ has demonstrated that the limbic system and the prefrontal cortex regions are involved in chronic alcohol addiction (Volkow et al., 2006). The limbic system is the major deriving force for the self-administration of psychoactive drugs, whereas the orbitofrontal cortex is the substrate of inhibition, involved in the termination of the inhibitory signal as shown in Figure 3. The neurobiology of addiction depends on personal preferences, including low-risk life style and high-risk lifestyle. Low-risk lifestyle is associated with non-addictive behavior, the tendency to prevent disease and promote health, and preference to live a healthy lifestyle. In contrast, a high-risk lifestyle is associated with cigarette smoking, binge alcohol drinking, chronic use of illicit drugs of abuse and addictive behavior associated with an unhealthy lifestyle, including improper dietary and sleep habits, which may lead to early morbidity and mortality as illustrated in Figure 4. In general craving can be broadly classified into two major classes: 1) craving for survival (also named as metabolic craving) including Kwashiorkor due to protein-calorie malnutrition, and 2) Marasmus due to inadequate energy intake of all forms. In some children, mixed clinical symptoms of Kwashiorkor and Marasmus are noticed. In all these metabolic disorders, there is a craving for the survival as presented in Figure 5. The second type of craving is to seek pleasure irrespective of potentially deleterious consequences.

\section{Desire for Self-Destruction}

This complex situation occurs mostly during adolescent age when a surge of secondary sexual characters occurs in both males and females. This situation occurs primarily due to psychoneuroendocrinological imbalance in the pituitary thyroid adrenal axis. These are mainly: growth hormone $(\mathrm{GH})$, follicle stimulating hormone (FSH), adrenocorticotropic hormone (ACTH), luteinizing hormone (LH), corticosteroids (Cortisol), the sex hormones estrogen and testosterone, and lastly the hormones of the thyroid gland including tri-iodothyronine $\left(\mathrm{T}_{3}\right)$, tetraiodo- 
Neurobiology of Addiction

\section{Limbic System}
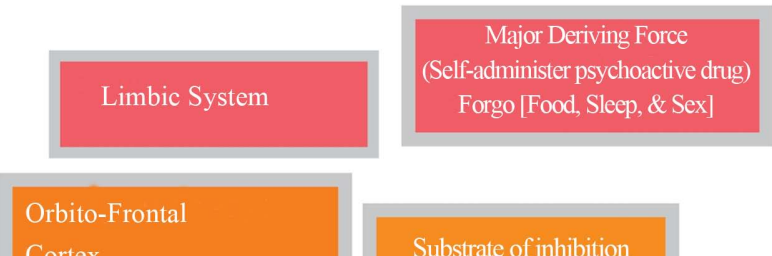

Cortex

Figure 3. A diagram illustrating the neurobiology of drug addiction involving primarily two brain regions: the prefrontal cortex and limbic system.

Neurobiology of Addiction

(Personal Preference)

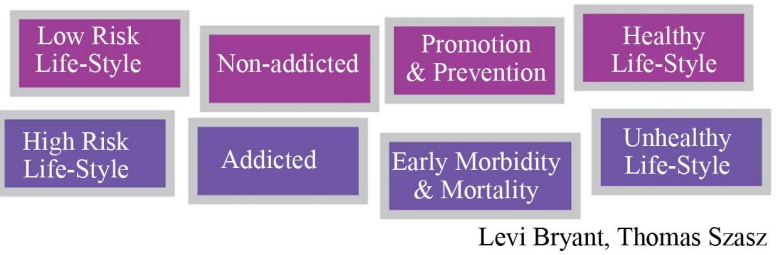

Figure 4. A diagram illustrating low risk or high risk personal preferences.
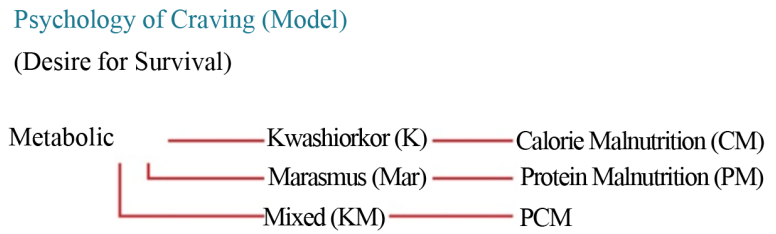

Figure 5. A diagram illustrating the psychology of craving: Desire for survival with metabolic consequences (Kwashiorkor, Marasmus or a patient with mixed Kwashiorkor and Marasmus symptoms). In these victims there is a craving for survival due to metabolic demand of protein-calories or both.

thyronine $\left(\mathrm{T}_{4}\right)$, thyroid stimulating hormone (TSH), and thyrotropin releasing hormone (TRH) at the hypothalamic level. Drunk-driving, over-speeding, and reckless behavior are some of the typical neurobehavioral abnormalities that are noticed among young adolescents due to psychoneuroendocrinological imbalance as we have recently reported [6]. Anorexia nervosa is characterized by emotional (limbic) starvation and leads to life-threatening food aversion. These patients have aversion to even intravenous (IV) dextrose saline. They can remove IV drips and escape from the hospital wards if not properly monitored. Anorexia nervosa patients develop senselessfood aversion and prefer to remain starved even if they are reduced to skeleton and near the verge of death. Such types of patients are difficult to clinically manage as they have a compromised immune system and are more prone to bacterial, viral and fungal infections. To clinically manage anorexia nervosa patients, $\gamma$-sterilized food at cryogenic temperature (Radapertized, radurized) may be provided to boost their immune system and enhance their desire for healthy food intake. The radurized and/or radapertized food can also be prescribed to chronic leukemia, post-war victims, chronic sick patients, and patients with chronic multiple drug abuse. Various antidepressants like selective serotonin reuptake inhibitors (SSRIs) like Fluoxetine can also reduce food intake, thus reducing hunger in the beginning and subsequently hypophagia to reduce food intake and weight reduction. Thus young adolescent girls may suffer from anorexia nervosa associated with food aversion, or on the other extreme bulimia, associated with food addiction as illustrated in Figure 6.

Undernutrition is most common among subjects trapped in a vicious circle of craving for food (usually carbohydrate, fat, and salt-rich), sex, and drug addiction to gain an antidepressant effect. Carbohydrate-rich diet enhances insulin secretion, which acts as a carrier for the essential amino acid, tryptophan in the CNS for the synthesis of serotonin. Brain regional depletion of serotonin is one of the major causes of depression. Tryptophan is converted to 5-hydroxy tryptophan by an enzyme, tryptophan hydroxylase, and 5-hydroxy tryptophan is converted to 5-hydroxy tryptamine (5-HT) in the presence of tryptophan decarboxylase and the coenzyme pyridoxal phosphate (PLP). So vitamin $\mathrm{B}_{6}$ supplementation and a tryptophan-rich diet can alleviate major depressive symptoms among patients with multiple drug addiction as $>40 \%$ patients suffering from craving for food, sex, and drugs of abuse may also present with major depressive symptoms. In addition, patients suffering from cocaine, methamphetamine, 3,4-methylenedioxy-N-methylamphetamine (MDMA) and heroin addiction are generally undernourished and immunocompromized, with psychotic symptoms, attention deficit hyperactivity disorder (ADHD), and reduced libido. Astronauts, postoperative patients, chronic cancer patients, people with

(Desire for Shelf-Destruction)

Psychoneuroendocrinological

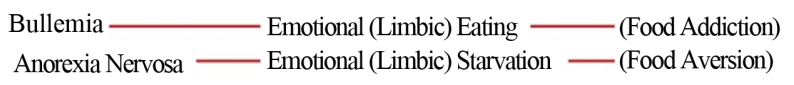

Figure 6. A diagram illustrating craving for self-destruction due to psychoneuroendocrinological disturbance in the pituitary thyroid adrenal axis. These two conditions are represented by Anorexia nervosa involving food aversion, whereas Bulimia is accompanied by excessive food addiction and even binge eating and drinking. Anorexia nervosa is life threatening. Both these clinical conditions are noticed among young adolescent girls during puberty when they develop secondary sexual characteristics. 
rheumatoid arthritis, HIV/AIDs patients, war-wounded soldiers with post-traumatic stress disorder, postpartum women, and several other groups of people with clinical conditions may also suffer from major depression beyond the scope of this article [7]. These patients also have special dietary requirements which needs proper sterilization, appetite control, and the appropriate nutritional value as they have a compromised neuroendocrine and immune system as illustrated in Figure 7.

\section{Experimental Studies}

To further elucidate the basic molecular mechanism of craving in drug addiction, we used homozygous weaver mutant (wv/wv) mice as a chronic model of drug addiction as these genotypes exhibit progressive neurodegeneration in the striatal dopaminergic neurons, hippocampal CA-3 and dentate gyrus regions, and cerebellar Purkinje cell layer as noticed in patients with multiple drug abuse. The distribution kinetics of ${ }^{18} \mathrm{~F}$-DOPA was significantly impaired in these genotypes and they exhibited progressive reduction in the striatal ${ }^{18} \mathrm{~F}$-DOPA uptake, indicating the involvement of impaired dopaminergic neurotransmission in drug addiction [8-10]. Chronic treatment with cocaine and methamphetamine significantly reduced striatal ${ }^{18} \mathrm{~F}$-DOPA as well as ${ }^{18} \mathrm{FdG}$ uptake. A further reduction in the striatal ${ }^{18} \mathrm{~F}$-DOPA and ${ }^{18} \mathrm{FdG}$ was noticed when cocaine and methamphetamine were administered along with ethanol, indicating that ethanol augments cocaine and methamphetamine addiction and could serve as a gateway to multiple drug abuse [11].

\section{Therapeutic Potential of Metallothioneins}

It is known that metallothioneins (MTs) are induced in drug addiction. Chronic nutritional stress, psychological stress (including hurries, worries, and curries) can induce poor nutritional status, malnutrition, malabsorption, zinc depletion, and neurodegeneration. During adverse stressful conditions of nutritional and psychological stress,

\begin{tabular}{|c|c|c|c|}
\hline \multicolumn{4}{|l|}{ Clinical Conditions } \\
\hline \multicolumn{4}{|l|}{ Undernutrition: } \\
\hline IMMUNOCOMPROMIZED C & CHRONIC DRUG ADDICTS & PSYCHOTIC & ADHD \\
\hline $\begin{array}{l}\text { PRE \& POST MENSTRUAL } \\
\text { SYNDROME }\end{array}$ & CANCER PATIENTS REDUC & JCED LIBIDO & ASTRONAUTS \\
\hline $\begin{array}{c}\text { POST-OPERATIONAL } \\
\text { PATIENTS }\end{array}$ & \multirow{2}{*}{$\begin{array}{l}\text { WAR-WOUNDED SOLDIERS } \\
\text { WITH POST-TRAUMATIC } \\
\text { STRESS DISORDER }\end{array}$} & \multirow{2}{*}{\multicolumn{2}{|c|}{$\begin{array}{l}\text { POST-PARTUM WOMEN } \\
\text { AND SEVERAL OTHER } \\
\text { CLINICAL CONDITIONS }\end{array}$}} \\
\hline HIV/AIDs PATIENTS & & & \\
\hline
\end{tabular}

[Special Dietary Requirements]

[Radapertized, Radurized] - (Gamma-Sterilized Food at Cryogenic Temperature)

Figure 7. A diagram illustrating various clinical conditions of under-nutrition where these patients may have special dietary requirements due to immune-compromise. These patients may require $\boldsymbol{\gamma}$-sterilized food at cryogenic temperature to avoid any chances of infection as they are highly prone to bacteria, viral and fungal infections. metallothioneins are induced in the CNS to provide neuroprotection. Zinc depletion inhibits autophagy of toxic proteins such as amyloid- $\beta$ which enhances further neurodegeneration, early morbidity and mortality. Zinc promotes autophagy and clears the aging brain from the accumulation of toxic proteinaceous materials responsible for progressive neurodegeneration. We discovered Charnoly body (CB) formation in the developing undernourished Purkinje neurons of the rat brain due to free radical overproduction and zinc depletion, which induces mitochondrial degeneration. The accumulation of mitochondrial membranes form electron-dense multilemellar structures that are resistant to autophagy and trigger apoptosis as presented in Figure 8. Thus CB formation serves as an early and sensitive biomarker of neurodegeneration. Metallothioneins (MTs) inhibit CB formation by acting as free radical scavengers as we have recently reported [12, 13]. Charnoly body formation can be prevented by nutritional rehabilitation and zinc repletion which facilitates neuroregeneration at physiological doses and neurodegeneration at toxic doses.

The exact role of CB formation in addiction remains unknown. A detailed study is required to further explain the intricate relationship between diet, sex, sleep, and drugs to elucidate the pathophysiological role various neurotransmitters, hormones, and their receptors to determine the exact psychology of craving. These studies may provide the judicious use of antipsychotic drugs including Risperidone, Domperidone, Quitiapine, Olanzapine, and Clonazepine for the clinical management of schizophrenia in addition to proper use of various antioxidants, as we recently reported, for progressive neurodegenerative disorders [14]. In addition, in-vitro experiments, in-vivo multimodality fusion neuroimaging employing CT/MRI/PET, and microarray analysis using

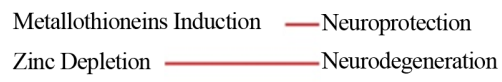

[Mesolimbic DA-ergic System]

Sex Addiction

Drug Addiction

Diet and Desire

Figure 8. A diagram illustrating zinc depletion causing neurodegeneration whereas metallothioneins (MTs) may provide neuroprotection. Metallothioneins inhibit Charnoly body formation by serving as free radical scavengers to provide mitochondrial neuroprotection. MTs are also induced during nutritional stress and drug addiction. The exact role of Charnoly body formation in drug addiction is yet to be established. 
omics biotechnology may provide detailed information regarding the psychological craving and its effective clinical management.

\section{Conclusion}

It is known that $70 \%-80 \%$ individuals with cravings for drug abuse may develop schizophrenia associated with visual and auditory hallucinations if it remains untreated. Although mesolimbic dopaminergic overload is primarily involved, other neurotransmitter systems including acetylcholine, serotonin, endorphins, and encephalins may also contribute to craving. Hence, in addition to psychotherapy, specific antidotes including Disulfiram for alcoholism; Nalaxone and Naltrexone for Opioids; and Methadone for Cocaine and Methamphetamine may provide beneficial effects to overcome cravings for drug abuse. Further developments in the novel discovery of specific antidotes will go a long way in the clinical management of cravings for food, sex, and drugs of abuse.

\section{Acknowledgements}

The authors express sincere thanks to Kallol Guha, President, Saint James School of Medicine, Anguilla for his encouragement and moral support.

\section{Conflict of Interests}

There is no conflict of interests for this article.

\section{REFERENCES}

[1] L. G. Costa, M. Aschner, A. Vitalone, T. Syversen and O. P. Soldin, "Developmental Neuropathology of Environmental Agents,” Annual Review of Pharmacology and Toxicology, Vol. 44, No. 1, 2004, pp. 87-110. http://dx.doi.org/10.1146/annurev.pharmtox.44.101802.1 $\underline{21424}$

[2] N. D. Volkow, G. H. Wang and D. Franceschi, et al., "Low Doses of Alcohol Substantially Decrease Glucose Metabolism in the Human Brain,” Neuroimage, Vol. 29, No. 1, 2006, pp. 295-301. http://dx.doi.org/10.1016/j.neuroimage.2005.07.004

[3] Anon, "Radiation Processing for Safe, Shelf-Stable and Ready-to-Eat Food," Proceedings of a Final Research Co-Ordination Meeting, Montreal, 10-14 July 2000, International Atomic Energy Agency, Geneva, 2003.
[4] “Alcohol Use in Australia. Issues and Stragtegies,” Department of Health, 2003-2004.

[5] R. J. Wurtman and J. J. Wurtman, "Brain Serotonin, Carbohydrate-Craving, Obesity and Depression," Obesity Research, Vol. 3, No. S4, 1995, pp. 477S-480S. http://dx.doi.org/10.1002/j.1550-8528.1995.tb00215.x

[6] M. Aarin, M. Haque, L. Johal, P. Mathur, W. Nel, A. Rais, R. Sandhu and S. Sharma, "Maturation of the Adolescent Brain,” Neuropsychiatric Disease and Treatment, Vol. 9, 2013, pp. 449-461.

[7] S. Faisal, A. Patel, C. Mattison, S. Bose, R. Krishnamohan, E. Sweeney, W. Nel, A. Rais, R. Sandhu, N. Ngu and S. Sharma, "Effect of Diet on Serotonergic Neurotransmission in Depression," Neurochemistry International, Vol. 62, No. 3, pp.324-329.

[8] S. Sharma and M. Ebadi, "Distribution Kinetics of ${ }^{18} \mathrm{~F}$ DOPA in Weaver Mutant Mice," Molecular Brain Research, Vol. 139, No. 1, 2005, pp. 23-30. http://dx.doi.org/10.1016/j.molbrainres.2005.05.018

[9] S. Sharma, H. El Refaey and M. Ebadi, "Complex-1 Activity and ${ }^{18}$ F-DOPA Uptake in Genetically Engineered Mouse Model of Parkinson's Disease and the Neuroprotective Role of Coenzyme $\mathrm{Q}_{10}$," Brain Research Bulletin, Vol. 70, No. 1, 2006, pp. 22-32. http://dx.doi.org/10.1016/j.brainresbull.2005.11.019

[10] M. Ebadi and S. Sharma, "Metallothioneins 1 and 2 Attenuate Peroxynitrite-Induced Oxidative Stress in Parkinson's Disease,” Experimental Biology and Medicine, Vol. 231, No. 9, 2006, pp. 1576-1583.

[11] S. Sharma and M. Ebadi, "SPECT Neuroimaging in Translational Research of CNS Disorders," Neurochemistry International, Vol. 52, No. 3, 2008, pp. 352-362. http://dx.doi.org/10.1016/j.neuint.2007.08.011

[12] S. Sharma, A. Rais, R. Sandhu, W. Nel and M. Ebadi, "Clinical Significance of Metallothioneins in Cell Therapy and Nanomedicine," International Journal of Nanomedicine, Vol. 8, No. 1, 2013, pp. 1477-1488. http://dx.doi.org/10.2147/IJN.S42019

[13] S. Sharma, C. S. Moon, A. Khogali, A. Haidous, A. Chabenne, C. Ojo, M. Jelebinkov, Y. Kurdi and M. Ebadi, "Biomarkers of Parkinson's Disease (Recent Update)," Neurochemistry International, Vol. 63, No. 3, 2013, pp. 201-222. http://dx.doi.org/10.1016/j.neuint.2013.06.005

[14] A. Sharma, M. Ebadi, S. Sharma and M. Ebadi, "Antioxidant Targeting in Neurodegenerative Disorders," In: I. Laher, Ed., System Biology of Free Radicals and Antioxidants, Springer-Verlag, Germany, 2013, pp. 1-30. 


\section{Highlights}

- Metallothioneins (MTs) are induced in nutritional stress and drug addiction.

- MTs inhibit Charnoly body (CB) formation by serving as free radical scavengers.

- Physical, psychological, and nutritional rehabilitation may inhibit CB formation.

- Nutritional rehabilitation facilitates Zinc-induced autophagy in the CNS.

\section{Abbreviations}

CB: Charnoly body, METH: Methamphetamine,

MDMA: Methylene deoxy methamphetamine, MTs: Metallothioneins. 